\section{Spectral Sensitivity of Single Visual Cells}

Since the work of Young and Helmholtz, the highly developed colour vision in man and many animals has been thought to depend on several, usually three, retinal receptors with different spectral sensitivity ${ }^{1,2}$. Such a theory is best suited for the explanation of human sensations, of animal reactions, and of electrical responses of the whole eye. It is, however, very difficult to determine the spectral sensitivity of the postulated single receptors using these methods. The existence of different photosensitive pigments has been demonstrated by chemical methods in vertebrates ${ }^{3}$, and in vivo photometric measurements have shown them for the mammalian, retina ${ }^{4}$. But this again does not give us an entirely satisfactory explanation of the principal unit-level functions, and the very successful chemical analyses of the visual pigments do not help us understand colour vision ${ }^{5}$.

Hitherto, electrical recordings from single elements in the vertebrate retina have been carried out only on the level of ganglion cells and not on the level of single receptors ${ }^{5,6}$. It is clear from these experiments that in the layer of bipolar cells and in the inner plexiform layer we are dealing with the integrated activity and not with the primary reactions of visual cells. Electrical recording from single visual cells of the blowfly (Calliphora erythrocephala) was made successfully, using microelectrodes with a tip diameter of approximately $0 \cdot 1 \mu$ (ref. 7 ).

On the basis of results of electrophysiological and behavioural experiments, colour vision appears to exist in Calliphora, and the spectral sensitivity of the whole eye is well known ${ }^{8}$. The study of the spectral sensitivity of single visual cells in this animal may provide information necessary for the understanding of colour vision in arthropods and might probably provide a model for colour vision in general. Basic properties of the receptor potentials, the mechanism of analysing the plane of polarized light in the ommatidia, and details of the methods are described elsewhere ${ }^{7,8}$

In the present experiments, stimulating lights were monochromatic flashes of equal quanta and of 0.1 or 0.02 sec. duration with an interval of 10 sec. The spectral range 315-681 $\mathrm{m} \mu$ was covered by a series of narrow-band interference filters with a mean wave-length interval of $19 \mathrm{mu}$ between filter peaks.

The spectral sensitivity of 39 single visual cells was determined for the wild form of Calliphora ; additional experiments were performed with the white-apricot mutant, in which the red screening pigment is absent.

All cells in the dorsal part of the eye have a uniform sensitivity curve with on $\Theta$ maximum at $489 \mathrm{~m} \mu, \sigma=$ $\pm 7 \mathrm{~m} \mu$, and a second maximum in the ultra-violet at $345 \mathrm{~m} \mu, \sigma= \pm 3 \mathrm{~m} \mu$. Three different receptor types were found in the ventral part of the eye: (1) a green receptor similar to that in the dorsal part with on $\theta$ maximum at $491 \mathrm{~m} \mu, \sigma= \pm 6 \mathrm{~m} \mu$, and a second maximum in the ultra-violet, again at $345 \mathrm{~m} \mu, \sigma= \pm$ $1 \mathrm{~m} \mu$; (2) a blue receptor with one maximum in the visible part of the spectrum at $468 \mathrm{~m} \mu, \sigma= \pm 6 \mathrm{~m} \mu$, and a second maximum again at $345 \mathrm{~m} \mu, \sigma= \pm 2 \mathrm{~m} \mu$ in the ultra-violet; (3) a yellow-green receptor with one maximum at $524 \mathrm{~m} \mu, \sigma= \pm 7 \mathrm{~m} \mu$, and another maximum also at $345 \mathrm{mu}$.

Statistical treatment for the 491-m $\mu$ and 468-mu peaks gave $P_{20}<0.0002$; for the $491-\mathrm{m} \mu$ and for the 524-m $\mu$ peaks, $P_{19}<0.0002$; for the $468-\mathrm{m} \mu$ and 524-m $\mu$ peaks, $P_{5}=0.0002$. Comparison of the maximum for the dorsal part at $489 \mathrm{~m} \mu$ with the maximum for the ventral part at $491 \mathrm{~m} \mu$ gives $P=0.8$. All maxima in the ultra-violet are very close to each other, with $P^{\prime}$ between 0.48 and 0.66 .

If one compares sensitivity curves of receptors belonging to the most common type with the 491-m $\mu$ maximum by setting the maximum at $491 \mathrm{~m} \mu$ as 100 per cent amplitude, the curves follow one another with one exception; some of them have a hump or even a third peak at $616 \mathrm{~m} \mu$. If the elements are compared according to the absolute value of potential amplitudes, the following rule becomes clear : the lower the response of an element, the more significant becomes the hump at $616 \mathrm{~m} \mu$, and only elements with very feeble action potentials show a peak at $616 \mathrm{~m} \mu$. This observation shows that the red screening pigment does not absorb light of this wave-length, and in weakly illuminated elements the portion of scattered. red light becomes much greater than the portion of light coming through the dioptric apparatus of the ommatidium. On the other hand, except in the case of weakly illuminated elements and in the far red, the screening pigments are not relevant to the shape of the sensitivity curves. It appears from these experiments that Calliphora erythrocephala has at least three visual receptors with different spectral sensitivities. All sensitivity curves have a uniform peak at $345 \mathrm{~m} \mu$. This corresponds very well to the rather stable position of the $\beta$-absorption in all derivatives of vertebrate visual pigments. The correct value of this wavelength may be the same in Calliphora and in vertebrates, because our measurements may have been affected by the quality of filters available. It may be assumed that the visual pigment of the most common type of visual cell in Calliphora is a retinene with the maximum absorption near $490 \mathrm{~m} \mu$. This pigment is already known to occur in insects ${ }^{10}$.

All the cells in the upper part of the eye yielded only one uniform curve. This situation is similar to that in other insects, which have been found to have one part of the eye sensitive to colours and another part colour-blind; for example, Periplaneta ${ }^{11}$, Notonecta ${ }^{12}$, and Libellula ${ }^{13}$. The most common receptor in the ventral part functions like the dorsal one. The numerical relationship among green, blue and yellow-green receptors is $18: 4: 3$. If the assumption is made that five of the seven visual cells within each ommatidium are green receptors, and, of the remaining two, one is a blue and the other a yellowgreen receptor, the numerical relationship would come very close to that actually found.

Department of Zoology,

H. Autrum University of Munich.

${ }^{1}$ Brindley, G. S., Progress in Biophysics, 8, 49 (1957).

${ }^{2}$ Daumer, K., Z, vergl. Physiol., 38, 413 (1956).

${ }^{3}$ Wald, G., Handbook of Physiology, Sect. 1, Neurophysiology, 1, 671 (1959).

' Rushton, W. A. H., Nat. Phys. Lab., Teddington, Paper 1 (1957), Weale, R. A., Brit. J. Ophthal., 4i, 461 (1957).

sutrum, H., Fortschr. Zool., N.F., 12, 176 (1960).

MacNichol, E. J., and Svaetichin, G., Amer. J. Ophthal., 46, 26 (1958).

7 Burkhardt, D., and Autrum, H., Z. Natforsch., 15b, 612 (1960).

'Autrum, H., and Stumpf, Hildegard, $Z$. vergl. Physiol., 35, 71 (1953). Schneider, G., ibid., 39, 1 (1956). Walther, J. B., and Dodt E., $Z$. Natforsch,,14b, 273 (1959). Autrum, H., and Hoffmann, Ch.
(unpublished work).

- Burkhardt, D., and Wendler, Lotte, Z. vergl. Physiol., 43, 687 (1960).

${ }^{10}$ Goldsmith, T. H., Ann. New York Acad. Sci., 74, 223 (1958).

11 Walther, J. B., J. Insect Physiol., 2, 142 (1958)

12 Rokohl, R., Z. vergl. Physiol., 29, 638 (1942).

${ }_{13}$ Mazokhin-Porshnyakov, G. A., Akad. Nauk SSSR., Biofizika, 4, 427 (1959). 